\title{
Higher Order Chromatic Aberration of Betatron Function of Circular Accelerators
}

\begin{abstract}
M. Takao*, K. Soutome, H. Tanaka, SPring- 8 , Abstract

The second order formula for the chromatic aberration of the betatron function of a circular accelerator is derived by means of the perturbative method. We confirm the validity of the formula for the chromatic aberration of the betatron function by comparing the numerical calculation with the measurement at the SPring-8 storage ring.
\end{abstract}

\section{INTRODUCTION}

The chromaticity control is very important ingredient in the operation of a high energy ring accelerator. The strong nonlinear magnetic field is then necessary to correct the large chromaticity, which inevitably enhances the nonlinearity of the beam dynamics of the circular accelerator. Hence, for the purpose of precisely controlling the chromaticity, one should understand the higher order behavior with respect to the momentum deviation.

In the preceding paper [1] we derived the higher order formula of the nonlinear dispersion function of a circular accelerator by means of the perturbative method. Based on the formulation the perturbative formula of the nonlinear chromaticity is derived in [2]. In these proceedings we develop the formulation of the chromaticity to the local one, i.e. the chromatic aberration of the betatron function. Using the local information of the chromaticity, one can control the global one in detail over the wide momentum range.

\section{FORMULATION}

\subsection{Hamiltonian}

We describe the motion of a particle in a ring accelerator by the following Hamiltonian $H$ [1]

$$
\begin{aligned}
& H\left(x, p_{x}, y, p_{y}\right) \\
& =-\left(1+K_{x} x\right) \sqrt{(1+\delta)^{2}-p_{x}^{2}-p_{y}^{2}}+\frac{1}{2}\left(1+K_{x} x\right)^{2} \\
& +\sum_{n=0} \frac{g_{n}}{(n+2) !} \sum_{m=0}^{\left[\frac{n}{2}\right]+1}(-)^{m}\left(\begin{array}{c}
n+2 \\
m
\end{array}\right) x^{n+2-2 m} y^{2 m}(1)
\end{aligned}
$$

where $\delta$ is the fractional deviation of the momentum $\delta=$ $\left(p-p_{0}\right) / p_{0}$ with the nominal momentum $p_{0}, K_{x}$ is the horizontal curvature, and $g_{n}$ 's are the strengths of multipole magnets.

To investigate the betatron oscillation around the offmomentum trajectory, we expand the Hamiltonian with respect to the momentum deviation $\delta$. Since the offmomentum trajectory $\left(x_{\epsilon}, p_{\epsilon}\right)$ is expressed by the dispersion function and its derivative whose perturbative formula

*takao@spring8.or.jp is given in [1], the Hamiltonian descib
tion with momentum deviation $\delta$ is

$$
\begin{aligned}
H_{\delta}= & \frac{1+K_{x} x_{\epsilon}}{2 \sqrt{(1+\delta)^{2}-p_{\epsilon}^{2}}}\left[\frac{(1+\delta)^{2}}{(1+\delta)^{2}-p_{\epsilon}^{2}} p_{x}^{2}+p_{y}^{2}\right] \\
& +\frac{K_{x} p_{\epsilon}}{\sqrt{(1+\delta)^{2}-p_{\epsilon}^{2}}} x p_{x} \\
& +\frac{1}{2} K_{x}^{2} x^{2}+\frac{1}{2}\left(\sum_{n=0} \frac{g_{n}}{n !} x_{\epsilon}^{n}\right)\left(x^{2}-y^{2}\right) .
\end{aligned}
$$

\subsection{Equation of Motion and Transfer Matrix}

In [2] we show that the equation of motion of an offmomentum particle derived from the Hamiltonian (2) becomes the following Hill's equation

$$
\left(\begin{array}{c}
z^{\prime} \\
p_{z}^{\prime}
\end{array}\right)=\left(\begin{array}{cc}
0 & 1 \\
-G_{z} & 0
\end{array}\right)\left(\begin{array}{c}
z \\
p_{z}
\end{array}\right) \equiv \mathbf{T}_{z}\left(\begin{array}{c}
z \\
p_{z}
\end{array}\right),
$$

where

$G_{z}=B_{z} C_{z}-A_{z}^{\prime}+\frac{\left(\log B_{z}\right)^{\prime \prime}}{2}-\left[A_{z}-\frac{\left(\log B_{z}\right)^{\prime}}{2}\right]^{2}$

with $z$ representing $x$ or $y$ and, e.g. for $z=x$

$$
\begin{aligned}
A_{x} & =\frac{K_{x} p_{\epsilon}}{\sqrt{(1+\delta)^{2}-p_{\epsilon}^{2}}}, \quad B_{x}=\frac{(1+\delta)^{2}\left(1+K_{x} x_{\epsilon}\right)}{\left[(1+\delta)^{2}-p_{\epsilon}^{2}\right]^{3 / 2}} \\
C_{x} & =-K_{x}^{2}-\sum_{n=0} \frac{g_{n}}{n !} x_{\epsilon}^{n} .
\end{aligned}
$$

The explicit forms of the some higher order terms of the perturbative expansion of $G_{z}$ with using the higher order dispersion function [1] are given in [2].

Since the motion of a particle is described by the Hill's equation, we can construct the transfer matrix in terms of 2 the Twiss parameter as usual. Hence the one turn transfer matrix over the circumference $L$ is given by

$$
\begin{aligned}
& \mathbf{M}_{z}\left(s_{0}+L \mid s_{0}\right) \\
& =\left(\begin{array}{cc}
\cos \mu_{z}+\alpha_{z} \sin \mu_{z} & \beta_{z} \sin \mu_{z} \\
-\gamma_{z} \sin \mu_{z} & \cos \mu_{z}-\alpha_{z} \sin \mu_{z}
\end{array}\right)
\end{aligned}
$$

where $\alpha_{z}, \beta_{z}$ and $\gamma_{z}$ are the Twiss parameters and $\mu_{z}$ the phase advance defined by

$$
\mu_{z}=\int_{s_{0}}^{s_{0}+L} \frac{d s}{\beta_{z}(s)} .
$$

In the previous paper [2], by expanding the transfer matrix $\mathbf{M}$ and the phase advance $\mu$ on $\delta$ as

$$
\mathbf{M}=\sum_{n=0} \delta^{n} \mathbf{M}_{n}, \quad \mu=\sum_{n=0} \delta^{n} \mu_{n}
$$


in the fundamental equation $\operatorname{Tr} \mathbf{M}\left(s_{0}+L \mid s_{0}\right)=2 \cos \mu$, we derive the perturbative formula for the global chromaticity. From now on, we omit the index denoting the coordinate $x$ or $y$ for the sake of simplicity.

\subsection{First Order Formula}

Before formulating the second order chromatic aberration of the betatron function, we reexamine the first order case in our formulation. Using the first order term of the impact transfer matrix $\mathbf{T}$, we obtain the $(1,2)$ component of the one turn matrix $\mathbf{M}$ at the first order

$$
\begin{aligned}
& \left.\mathbf{M}_{1}\left(s_{0}+L \mid s_{0}\right)\right|_{12} \\
& =\left.\int_{s_{0}}^{s_{0}+L} d s_{1} \mathbf{M}_{0}\left(s_{0}+L \mid s_{1}\right) \mathbf{T}_{1}\left(s_{1}\right) \mathbf{M}_{0}\left(s_{1} \mid s_{0}\right)\right|_{12} .
\end{aligned}
$$

On the other hand, from the $(1,2)$ component of the matrix (4), we obtain the representation in terms of the betatron function

$$
\left.\mathbf{M}_{1}\left(s_{0}+L \mid s_{0}\right)\right|_{12}=\beta_{1}\left(s_{0}\right) \sin \mu_{0}+\beta_{0}\left(s_{0}\right) \mu_{1} \cos \mu_{0},
$$

where $\beta_{1}$ denotes the first order modulation of the beta function $\beta=\sum_{n=0} \delta^{n} \beta_{n}$. The first order phase advance $\mu_{1}$ has been already known as given in [2, 3, 4]. Hence, equating the above equations and inserting the explicit form of $\mathbf{T}_{1}$ into the resultant, we find the integral formula for the linear chromatic aberration of the betatron function

$$
\begin{aligned}
\beta_{1}\left(s_{0}\right)=-\quad & \frac{\beta_{0}\left(s_{0}\right)}{2 \sin \mu_{0}} \int_{s_{0}}^{s_{0}+L} d s_{1} \beta_{0}\left(s_{1}\right) G_{1}\left(s_{1}\right) \\
& \times \cos \left[\mu_{0}-2 \varphi\left(s_{1}\right)+2 \varphi\left(s_{0}\right)\right] .
\end{aligned}
$$

The consistency of the first order formula of the betatron function with the expression of the global chromaticity is proven by the integration over the circumference. By definition, the first order global chromaticity $\mu_{1}$ should be represented by $\beta_{1}$ as

$$
\mu_{1}=-\int_{0}^{L} d s_{0} \frac{\beta_{1}\left(s_{0}\right)}{\beta_{0}^{2}\left(s_{0}\right)} .
$$

Integrating the right hand side of Eq. (7) with the representation (6), we find

$$
-\int_{0}^{L} d s_{0} \frac{\beta_{1}\left(s_{0}\right)}{\beta_{0}^{2}\left(s_{0}\right)}=\frac{1}{2} \int_{0}^{L} d s_{0} \beta_{0}\left(s_{0}\right) G_{1}\left(s_{0}\right),
$$

which exactly coincides with the expression of the first order chromaticity $\xi_{1}=\mu_{1} /(2 \pi)[2,3,4]$.

\subsection{Second Order Formula}

The second order term of the $(1,2)$ component of the one turn matrix $\mathbf{M}$ consists of the two contributions from the impact transfer matrix $\mathbf{T}$

$\left.\mathbf{M}_{2} \quad\left(s_{0}+L \mid s_{0}\right)\right|_{12}$

$$
\begin{gathered}
=\left.\int_{s_{0}}^{s_{0}+L} d s_{1} \mathbf{M}_{0}\left(s_{0}+L \mid s_{1}\right) \mathbf{T}_{2}\left(s_{1}\right) \mathbf{M}_{0}\left(s_{1} \mid s_{0}\right)\right|_{12} \\
+\int_{s_{0}}^{s_{0}+L} d s_{2} \int_{s_{0}}^{s_{2}} d s_{1} \mathbf{M}_{0}\left(s_{0}+L \mid s_{2}\right) \mathbf{T}_{1}\left(s_{2}\right) \\
\quad \times\left.\mathbf{M}_{0}\left(s_{2} \mid s_{1}\right) \mathbf{T}_{1}\left(s_{1}\right) \mathbf{M}_{0}\left(s_{1} \mid s_{0}\right)\right|_{12} .
\end{gathered}
$$

The former term corresponds to the second order modulation of the Hamiltonian and the latter indicates the cross term of the first order impact.

On the other hands, the $(1,2)$ component of the one turn matrix $\mathbf{M}$ is also represented in terms of the higher order terms of the betatron function as

$$
\begin{gathered}
\left.\mathbf{M}_{2}\left(s_{0}+L \mid s_{0}\right)\right|_{12}=\beta_{2}\left(s_{0}\right) \sin \mu_{0}+\beta_{1}\left(s_{0}\right) \mu_{1} \cos \mu_{0} \\
+\beta_{0}\left(s_{0}\right)\left(\mu_{2} \cos \mu_{0}-\frac{1}{2} \mu_{1}^{2} \sin \mu_{0}\right) .
\end{gathered}
$$

Using the formula for the higher order phase advances $\mu_{1,2}$ given in [2], we can obtain the representation of the second order aberration of the betatron function $\beta_{2}$

$$
\begin{aligned}
\beta_{2}\left(s_{0}\right)=- & \frac{\beta_{0}\left(s_{0}\right)}{2 \sin \mu_{0}} \int_{s_{0}}^{s_{0}+L} d s_{1} \beta_{0}\left(s_{1}\right) G_{2}\left(s_{1}\right) \\
& \times \cos \left[\mu_{0}-2 \varphi\left(s_{1}\right)+2 \varphi\left(s_{0}\right)\right] \\
+ & \frac{\beta_{0}\left(s_{0}\right)}{4 \sin ^{2} \mu_{0}} \int_{s_{0}}^{s_{0}+L} d s_{2} \int_{s_{0}}^{s_{2}} d s_{1} \beta_{0}\left(s_{2}\right) G_{1}\left(s_{2}\right) \\
\times & \beta_{0}\left(s_{1}\right) G_{1}\left(s_{1}\right)\left\{\cos 2\left[\mu_{0}-\varphi\left(s_{2}\right)+\varphi\left(s_{0}\right)\right]\right. \\
+ & \left.\cos 2\left[\varphi\left(s_{2}\right)-\varphi\left(s_{1}\right)\right]+\cos 2\left[\varphi\left(s_{1}\right)-\varphi\left(s_{0}\right)\right]\right\} .
\end{aligned}
$$

In order to confirm the consistency of the present formula for the local chromaticity with the global one, we have to check the following defining equality

$$
\mu_{2}=-\int_{0}^{L} \frac{d s_{0}}{\beta_{0}\left(s_{0}\right)}\left[\frac{\beta_{2}\left(s_{0}\right)}{\beta_{0}\left(s_{0}\right)}-\left\{\frac{\beta_{1}\left(s_{0}\right)}{\beta_{0}\left(s_{0}\right)}\right\}^{2}\right] .
$$

After the lengthy calculation, one can convince oneself of the validity of these formula.

\section{NUMERICAL CALCULATION}

\subsection{Fourier Transform}

In investigating the higher order terms of the global chromaticity, we find that the Fourier transform with respect to a lattice period is an effective measure to numerically integrate the formula. For example, if defining the Fourier components of $\beta_{0} G_{1}$ for $n=0,1,2, \ldots$ as

$a_{1}(n)=\frac{2}{\mu_{0}} \int_{s_{0}}^{s_{0}+L} d s_{1} \cos \left[\frac{2 \pi n}{\mu_{0}} \varphi\left(s_{1}\right)\right] \beta\left(s_{1}\right) G_{1}\left(s_{1}\right)$ and $b_{1}(n)$ for $\sin \left[\frac{2 \pi n}{\mu_{0}} \varphi\left(s_{1}\right)\right]$, we have

$$
\begin{aligned}
& \beta_{1}\left(s_{0}\right)=-\frac{\beta_{0}\left(s_{0}\right)}{4}\left[a_{1}(0)+\sum_{n=1}^{\infty} \frac{2 \mu_{0}^{2}}{\mu_{0}^{2}-\pi^{2} n^{2}}\right. \\
& \left.\times\left\{a_{1}(n) \cos \frac{2 \pi n \varphi_{0}\left(s_{0}\right)}{\mu_{0}}+b_{1}(n) \sin \frac{2 \pi n \varphi_{0}\left(s_{0}\right)}{\mu_{0}}\right\}\right] .
\end{aligned}
$$


While checking the convergence, we sum up the Fourier series to the twenty thousand harmonics. It should be extremely emphasized that the thickness of the sextupole magnet plays an important role for the convergence of the Fourier series. Once the Fourier components are calculated, one can easily sum up the Fourier series at any point throughout the circumference.

The consistency of the formula for the local and global chromaticities can be easily verified in the frequency domain. The constant term of the chromatic aberration of the betatron function is just the global chromaticity, since the oscillating terms disappear after the integration over the circumference, i.e. one period.

\subsection{A Numerical Example}

As an example, we evaluate the chromatic aberration of the betatron function of the SPring- 8 storage ring. The storage ring is composed of 44 cells of double bend achromat and 4 magnet free straight sections of $30 \mathrm{~m}$ long, which eventually possesses fourfold symmetry. The horizontal betatron function is indicated in Fig. 1. Note that the storage ring is normally operated at $(40.15,18.35)$ in tune map. The modulation of the betatron function in the middle of Fig. 1 corresponds to the long straight section.

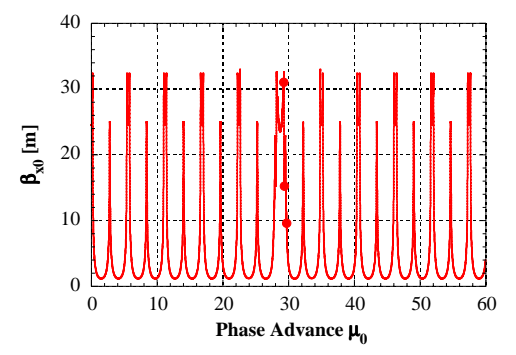

Figure 1: The horizontal betatron function of the SPring-8 storage ring over the quarter of the circumference.

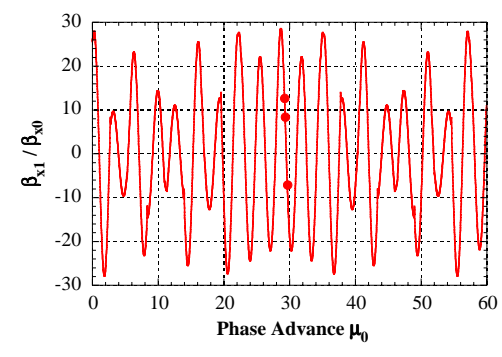

Figure 2: The first order aberration of the horizontal betatron function of the SPring- 8 storage ring.

The first and the second order aberration of the betatron function are shown in Figs. 2 and 3, respectively. The higher order aberrations, off course, possess the fourfold symmetry corresponding to the ring symmetry.

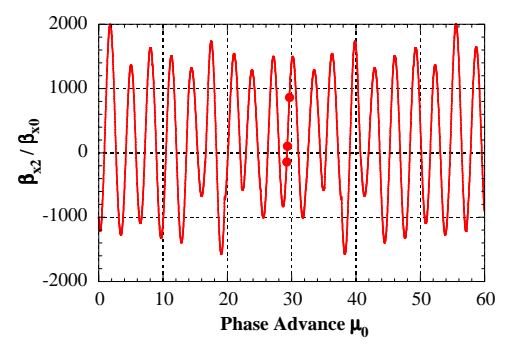

Figure 3: The second order aberration of the horizontal betatron function of the SPring- 8 storage ring.

\subsection{An Experiment}

The betatron function is measured by detecting the tune shift as the strength of the individual quadrupole magnet varied. Hence, measuring the betatron function for different $r f$ frequency, one can get the chromatic aberration.

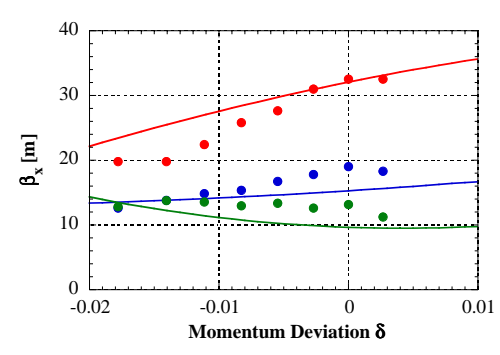

Figure 4: The measured and calculated horizontal betatron functions of the SPring- 8 storage ring as the momentum deviation varied. The full circles represent the measured betatron functions and the solid lines the calculated ones.

The measurement of the betatron function is performed at three different quadrupole magnets situated at downstream immediately after the long straight section, whose data are shown in Fig. 4 by the full circles. The calculated betatron functions and the higher order aberrations at the data points are displayed by the full circles in Figs. 1, 2 and 3 .

The calculated betatron functions up to the second order aberration are represented by the solid lines in Fig. 4 . Although the discrepancy between the measured and the calculated betatron functions is somewhat large, the tendencies of the functions well agree on the whole.

\section{REFERENCES}

[1] K. Soutome, M. Takao and H. Tanaka, in Proc. of 6th EPAC (Stockholm, 1998), 924; H. Tanaka, et al., Nucl. Instrum. Methods A 431 (1999), 396.

[2] M. Takao, K. Soutome and H. Tanaka, in Proc. of 7th EPAC (Vienna, 2000), 1083; M. Takao, et al., in preparation.

[3] M. Sands, in Proceedings of International School of Physics Enrico Fermi 46 (Academic Press, San Diego, 1971), 257.

[4] F. Willeke and G. Ripken, in AIP Conf. Proc. 184 (America Institute of Physics, New York, 1989), 758. 